\section{Maternal Mortality and Lead}

Important as are the effects of small doses of lead on health, they acquire added gravity as a possible factor in the causation of maternal mortality. The Registrar-General divides deaths in childbed into two groups: those from puerperal fever, and those due to "eclampsia and other accidents and diseases." Taking the country as a whole, puerperal fever is the most frequent cause of maternal deaths. In places supplied with soft moorland water the position is reversed. There, eclampsia and other accidents and diseases outnumber the deaths from puerperal fever, in some places by more than three times. In an appendix to her report on maternal mortality, Dame Janet Campbell gives the death rates of the Registrar-General's two groups for the four years 1919-22. During those four years the fewest maternal deaths from eclampsia and other accidents and diseases occurred in London, where they were 1.62 per 1,000 births. The London water, drawn largely from chalk-bearing strata, does not dissolve lead. In Dewsbury, Halifax, Huddersfield, Rochdale, and the county of Breconshire, supplied with plumbo-solvent water, the maternal mortality from the same causes was over 5 per 1,000 births, or more than three times that of London, while in Westmorland, Anglesey, Carmarthenshire, Caernarvonshire, Merionethshire, and Radnorshire, it was over 4 per 1,000 births, or about three times that of London. In the county of Westmorland, while there were three maternal deaths from puerperal fever, there were ten from eclampsia. The high maternal death rate from eclampsia and other accidents and diseases occurs where there is soft plumbosolvent water. The main responsibility for this high death rate rests on eclampsia.

There must be some reason for the disparity between the figures for London and the places I have quoted. Other accidents and diseases of childbed cannot be more frequent in the places I have named than in London. The disparity is greater than the figures indicate, for the I.ondon figures are swelled by the cases of eclampsia which occur there. We know that lead is eliminated by the kidneys, and though I can produce no analyses of either blood or urinary urea, the sluggishness and somnolence I have described are not unlike the defective kidney action of Bright's disease. In its grosser forms of poisoning lead damages the kidneys, and more than one of my patients suffering from the subtle form of plumbism have had the nephralgia characteristic of the passage of gravel through the urinary organs.

Next to septicaemia eclampsia is the highest factor in maternal mortality. Knowing the symptoms which I have felt in my own person and observed in my patients, and remembering the frequency of eclampsia when subtle lead poisoning was prevalent in Huddersfield, the high mortality from eclampsia and other accidents and diseases of pregnancy in places supplied with plumbo-solvent water suggests that the continuous ingestion of minute quantities of lead may be the explanation of an otherwise unaccountable mortality. Though the quantity of lead imbibed may be small, it may be enough to turn the scale and precipitate the occurrence of cclampsia in a pregnant woman on the verge of toxaemia. If the conclusions I draw from the figures I have quoted are well founded-and they are confirmed by an examination of the death rates in other places too numerous for mention here-we have, ready to our hands, one means of reducing a great cause of the baffling problem of maternal mortality.

The remedy is simple-to stop the ingestion of lead. This can be done by sending the patient away from the lead, by substituting pipes lined with block tin for the lead pipes, or by passing every, drop of water used in the preparation of food or for drinking purposes through a filter with a charcoal filtering bed. The so-called tinwashed pipes are not a protection, and only a filter with a charcoal filtering medium can be trusted to remove lead from a plumbo-solvent water.

Water supplies contain organic matter derived from the soil of the gathering ground, sometimes sufficient to discolour the water. In moorland waters there is an acid derived from the peat. The lead dissolved is not a simple inorganic salt, but a complex organic compound. It is by its affinity for organic substances that lead injures the body and destroys the embryo in utero. Charcoal, as we know from its effects in fermentative disorders of the digestive tract, attacks organic matter. In a carbon filter the fraction of organic lead in the water is acted upon by a mass of carbon compared with which a dose of charcoal by the mouth is insignificant. After from six to twelve months, according to the use it has had, the charcoal is not a reliable remover of the lead, and a fresh charge should be put into the filter.

No medicinal treatment is necessary to expel lead from the body. If the ingestion of lead is stopped and the eliminatory organs are active, the body will get rid of it. If iodides liberate lead from the tissues, the extra lead set free will aggravate the patient's symptoms.

\section{SOME NEGLECTED SYMPTOMS IN ACUTE ABDOMINAL DISEASE*}

$\mathrm{BY}$

\section{ZACHARY COPE, B.A., M.D., M.S., F.R.C.S} SURGEON TO ST, MARY'S AND THE BOLINGBROKE HOSPITALS

Abdominal disease, whether acute or chronic, is diagnosed by considering three groups of observations. In the first place, local anatomical changes are detected by inspecting with the eyes or $x$ rays, feeling with the fingers, or even by listening with the stethoscope; secondly, perversions of physiological function are deduced from certain symptoms indicative of visceral derangement or by carrying out certain tests which betray pathological changes in the abdominal organs; thirdly, when the disease is serious and is influencing the whole organism, note is taken of the great bodily systems, especially the circulatory, respiratory, metabolic, and excretory systems.

In chronic abdominal disease the slowness of the process allows time for compensation to occur ; the slight physiological changes are masked or neglected until gross anatomical alterations may attract the attention of the observer, or the general bodily depreciation may be obvious to the patient and his friends. In acute abdominal disease physiological derangement plays a much greater part ; rapid changes due to violent stimuli delivered without warning are the rule, and the need for immediate compensation of the vital systems is all too frequently unsatisfied; while gross anatomical changes either are absent or appear only at a comparatively late stage in the course of the illness. Unless these facts are appreciated diagnosis becomes haphazard and unscientific.

\section{Signs and Symptoms of Shock}

It is well, therefore, to consider in the first place that symptom which is fairly common but still misinterpreted and ill-understood-that is, shock. When a sudden internal haemorrhage occurs, or when a very violent visceral stimulus is applied, the direct or reflex disturbance of the circulation leads to that clinical picture which is generally known as shock-cold extremities, subnormal temperature, pallor, sweating, a rapid and small pulse, and a lowered blood pressure. With a person who has been in

* An address to the Norwich Division of the British Medical Association. 
good general health previously, however, compensation soon occurs, the circulation is soon re-established, and the observer finds it difficult to believe in the existence of any serious condition. Neglect to realize the wonderful compensatory mechanism of the circulation accounts for this misunderstanding.

Quite as serious is the failure to note minor degrees of shock. Shock is a clinical condition, and should not be defined too rigidly. When all the classical symptoms are present shock must be accounted extreme, but it may be present even when some of the symptoms are absent. Serious clinical shock may be present without pallor, without much increase in pulse rate, without much lowering of blood pressure; very seldom, however, does the temperature fail to be subnormal. Only when the diminution of blood volume becomes too great for the vasoconstriction to bring up the pressure to normal will the sphygmomanometer record a fall ; but before that stage is reached the smaller blood volume may lead to diminished metabolism, lowered temperature, sweating, and other general symptoms. It is well in acute cases to take the blood pressure several times. It will then be seen that it may fluctuate rapidly, and that one record may give misleading information. Instability of blood pressure is characteristic of those degrees of shock which fall short of the full picture.

In the case of a perforated ulcer of the stomach or duodenum a greater or less initial degree of shock is generally present, but compensation occurs inversely as the violence of the injurious stimulus. With a small leak shock is almost absent; with a large one it may be extreme, and go on to a fatal result; with intermediate cases it is moderate and fairly soon recovered from. Its occurrence is even denied by some authorities in all cases ; but a subnormal temperature, cold extremities, a sweating skin, and a pallid face are enough to diagnose clinical shock, and these are usually present for a time.

\section{Importance of Sphygmomanometry}

The estimation of the blood pressure is too frequently neglected in other cases. Particularly is this the case in intestinal obstruction. The constant vomiting and direct effect of the toxins lead to a great fall in blood pressure. Unless this is carefully measured the capacity of the patient to undergo any operative procedure may be woefully miscalculated. With a blood pressure under $100 \mathrm{~mm} . \mathrm{Hg}$ (systolic) it is seldom expedient to give a general anaesthetic. More important to recollect is the fact that the systolic pressure may be fairly high, but the difference between the systolic and diastolic small. This difference, representing the pulse-pressure which maintains the circulation, may be quite small, even though the systolic pressure may be approximately normal. I have recorded a case of intestinal obstruction in which the systolic pressure was 135 and the diastolic 110. This patient was in the last degree of collapse, and I gave it as my opinion that he would hardly stand enterostomy -an opinion which was unfortunately verified. You will note that the systolic pressure was maintained though the blood stream was dependent on a difference of pressure of $25 \mathrm{~mm}$. of mercury for its propulsion.

\section{PaIN}

When we come to consider the symptoms due to physiological derangements pain is the most important. Indeed, it is often possible to diagnose a case merely by considering the type, intensity, and position of the pain. Too often, however, pair is inquired after in a very general sort of way, and important points are omitted. I will give some examples.
In the first place, help is gained by noting the shifting of the position of the pain. The best and most common example is that of appendicitis. The initial epigastric or umbilical pain is followed by the complaint of pain or tenderness in the right iliac fossa. This sequence is nearly always found in a first attack, but may be and generally is absent in later attacks. With a retrocaecal position of the appendix the initial epigastric pain is often absent. The epigastric-right-iliac sequence in a young adult or child nearly always signifies appendicitis, but in older persons greater circumspection is needed in diagnosis. A leaking duodenal ulcer or an attack of acute pancreatitis may give rise to similar, though usually more severe, pains. The shifting of the pain with a perforated ulcer is usually characteristic. At first severe and epigastric, it is followed by general hypogastric pain and tenderness.

In cases of peritonitis arising within the pelvis diagnosis may be difficult at first, but the pain gradually shifts upwards as the inflammation ascends, and the distension of the bowel is noticed at a higher and higher level.

Severe loin pain due to passage of small stone or gravel down the ureter is frequently accompanied by pain felt in the iliac fossa. If there is doubt as to the diagnosis (that is, whether appendicitis is the cause) help is gained by noting if the site of the pain tends to move downwards, as it often does with ureteric colic.

In cases of pancreatitis the initial devastating pain is followed by a more lasting pain and tenderness in both loins. If this pain were unilateral renal inflammation would naturally be suspected, but taken along with the character of the initial pain the bilateral loin pain is almost diagnostic of acute pancreatitis. It is merely a local pain due to the reaction round the swollen tissues of the gland, which, as we know, spreads its tail and head into the left and right loin just below the level of the last ribs.

\section{Superficial Hyperaesthesia}

Superficial hyperaesthesia is often present in acute abdominal disease and is occasionally of considerable value in diagnosis. It is folly to attach too much importance to it, however, for the same area of hyperaesthesia can be produced by various intra-abdominal lesions. Yet every symptom is to be investigated, and I am sure that this is sometimes neglected. In young people the right iliac triangle is confirmatory of appendicitis, while in older persons it is only found with that disease or salpingitis. The bilateral triangle may accompany hypogastric peritonitis, and occasionally is found before any obvious peritonitis has been set up by an appendicitis.

The narrow band of hyperaesthesia above and parallel to Poupart's ligament is commonly present in subacute and subsiding appendicitis, but may also accompany pyelitis, salpingitis, or even cholecystitis and perforated duodenal ulcer. It is due to irritation of the parietal peritoneum in the corresponding iliac region. In any case of doubt between a thoracic and an abdominal lesion the finding of iliac hyperaesthesia weighs down the scale on the side of an abdominal lesion. This alone would make it worth while to test for hyperaesthesia in every case.

\section{Referred Pain}

Of much greater value in diagnosis is pain that is felt outside the abdomen. Most of these pains are well known, so that everyone is aware of the site of pain due to colic of the small intestine, large intestine, uterus, or renal pelvis. But it is not generally known that disease of the appendix may cause pain in the testicle either of the same or the opposite side. This is an added difficulty in differential diagnosis, and one that may and has led to mistakes. The referred pain of biliary colic is usually 
felt in the right subscapular region, but mistakes may result if one is ignorant of the fact that the pain may be felt anywhere in the zone of distribution of the eighth or ninth thoracic nerve. I have seen cases in which all the severe pain of biliary colic was felt in the left side of this zone.

\section{Phrenic Shoulder Pain}

That referred pain which $I$ have found of most use in difficult abdominal cases is phrenic pain. Though $I$ and a few others have been teaching the importance of this pain for several years the current textbooks make little or no mention of it, and certainly few make proper use of this symptom in their practice. I shall therefore dwell longer on this subject.

Phrenic referred pain is that pain which is felt on the top of the shoulder as the result of an irritative lesion in the region of the diaphragm. The phrenic nerve is made up of fibres from the third, fourth, and fifth cervical nerves, and the referred pain is felt in the region of distribution of the third and fourth, but chiefly the fourth, nerves. That area comprises the supraspinous fossa, the acromion and clavicular regions, and the subclavicular fossa. The loose way in which patients and doctors speak of the shoulder region when they actually mean the scapular or subscapular areas makes it necessary to specify top of the shoulder or tip of the shoulder pain.

The intensity and nature of the pain vary considerably. It may be so severe as to feel like a nail being driven in on top of the shoulder, or, on the other hand, may be so slight that the patient will not make any spontaneous complaint, and when asked about the pain will merely say that he had a pain in the shoulder which he put down to rheumatism. In a few cases the pain is felt in the deltoid region, and I have seen others where the patient stated that the muscles felt weak in that part.

Irritation of the right or left side of the diaphragm causes pain which is limited to the shoulder on the corresponding side; that is admitted by all. Inflammation in the middle part of the muscle may cause bilateral shoulder-top pain. In my experience there is also a further localizing value in this symptom, for $I$ have found it a general rule, with few exceptions, that an affection of the anterior or posterior part of the diaphragm causes pain on the front or back part of the shoulder. For example, irritation of the anterior fibres of the muscle will cause pain in the clavicular region, while an affection of the crura will lead to local pain in the supraspinous fossa. There are few exceptions to this important rule.

\section{Subdiaphragmatic Conditions}

Phrenic shoulder pain is of the utmost value in fixing the site of many acute abdominal disorders. Scarcely a week passes that I do not find it of the greatest assistance. In the last fortnight it clinched the diagnosis in two cases-a ruptured spleen and an acute pancreatitis. I will indicate the class of case in which it is generally or occasionally present. There are two groups which we must distinguish. First, those diseases which originate in organs which are in relation with the diaphragm. Secondly, those conditions which arise in other parts of the abdomen but may affect the diaphragm secondarily.

The first group comprises diseases of the liver and gallbladder, duodenum and stomach, spleen, pancreas, and, rarely, kidney. The most common disorder which gives rise to it is perforation of a gastric or duodenal ulcer. Phrenic pain occurs in about two out of every three such cases. In the case of duodenal ulcer the pain is most commonly felt in the right supraspinous fossa, as might be expected when we remember that the irritated part will be the right crus and contiguous part. Here I cannot help speculating on the reason for the origin of a curious error which has been and is being perpetuated from one textbook to another. It is stated with monotonous regularity that the pain of biliary colic is often felt on top of the right shoulder. Anyone who has observed his cases carefully over a number of years knows that, though such a reference of pain is possible, it is of the greatest rarity. Nearly every case of severe right hypochondriac pain associated with pain on top of the right shoulder is due to a leakage of a duodenal ulcer. Though I have known this for more than ten years yet on a few occasions I have discussed the diagnosis of a case with a practitioner and come to the conclusion that the rare exception of biliary colic with shoulder-top pain had at last turned up. In every case when I have operated later I have found a leaking ulcer. The writers who described shoulder-top pain in connexion with biliary colic put their views to paper before the surgery of acute abdominal disease had started, and I think that the error arose from mistaking the local leaking of an ulcer for gall-stone disease. If shoulder-top pain does occur with gall-stone disease it is almost always due to the onset of peritonitis which irritates the contiguous diaphragm.

With perforated gastric ulcer the pain is more often bilateral or felt on the left side. Occasionally it is limited to the clavicular region.

Tropical abscess of the liver is uncommon in this country, but phrenic shoulder pain is very common as an accompaniment of such an abscess. As a matter of interest it was through observing liver abscesses in Mesopotamia during the war that I was led to study the significance of pain referred from the diaphragm. When the symptom is present it signifies that the liver is adherent to the diaphragm and that the abscess is threatening to break through into the thorax. It is most often felt in the right supraspinous fossa, since abscesses usually affect the posterior part of the right lobe.

The occurrence of left shoulder-top pain in connexion with ruptured spleen is sometimes known as Kehr's sign. It is due to the irritation of the muscle caused by the effused blood.

Though it may not be accounted a surgical condition there is no harm in remembering that an infarct of the spleen may also be accompanied by phrenic shoulder pain.

Rarely acute pancreatitis may be accompanied by pain on top of the left shoulder localized to the supraspinous fossa. I have already mentioned a recent case that I have seen. I have seen one other similar case, and know of several more.

Very rarely perinephritis may irritate the diaphragm sufficiently to cause referred pain, but it is so rare and inconstant as to be of no practical value.

\section{Pelvic Conditions}

When we come to consider diseases originating away from the diaphragm the most important is ruptured ectopic pregnancy. The occurrence of shoulder-top pain in this condition has long been recognized, but its full significance is still far from being realized. The pain is due to the liquid blood flowing up to and irritating the diaphragm. It is a matter of chance whether the blood irritates the anterior or posterior part of the muscle, and so the position of the pain may be clavicular or supraspinous. There are few pathognomonic symptoms in surgery, but this can be regarded as a near approachthat is, severe collapse and hypogastric pain unattended by abdominal rigidity, but accompanied by pain on top of one or both shoulders, indicate a ruptured ectopic pregnancy or bleeding from a ruptured Graafian cyst. Even this well-established fact is not widely known. When peritonitis which has its origin in any part of the abrlomen reaches up to the diaphragm it may irritate sufficiently to cause shoulder pain. As a rule, if s!'ch a case comes 
to necropsy there will be found one or more plaques of plastic lymph between the liver and the diaphragm. This is more common on the anterior aspect, so the referred pain is more likely to be in the clavicular region.

I would not have anyone think that phrenic shoulder pain is ever to be relied upon to the exclusion of other symptoms, for the diaphragm has an upper as well as a lower surface, and thoracic disease may irritate this upper surface. That is only one more reason for making a thorough examination, so that no localizing sign may be omitted. It is worth mentioning that pain from diaphragmatic pleurisy is more commonly felt in the clavicular and subclavicular area.

\section{Muscular Rigidity}

I now come to the symptoms produced by the reflex rigidity of the muscles which bound the abdominal cavity. There should be little need to mention rigidity of the anterior and lateral abdominal muscles produced by peritonitis, since it is familiar to everyone who has seen half a dozen cases of acute abdominal disease. But it is not yet general knowledge-nay, it is often overlooked by the elect-that extensive peritonitis may exist without any rigidity at all.

At the risk of repeating what we all may know already I would point out that irritation of the internal lining of the abdominal cavity is responded to by a greater or lesser degree of rigidity of the muscles which wall in the cavity. There is a large area comprising the pelvic cavity and the median part of the posterior abdominal wall which is relatively insensitive or silent when stimulated, giving rise to no rigidity of the overlying or underlying muscles. It is very common to have early pelvic peritonitis without any muscular rigidity. Another fact which is overlooked is that old people with weak muscles and easily tired reflexes frequently show little or no rigidity with peritonitis.

Muscular rigidity of the deep-lying muscles is easily, and I think commonly, neglected. When the diaphragm is immobile on account of overlying inflammation we cannot always have an $x$-ray apparatus handy to show that it does not move on respiration, but we have an easy clinical test. If the abdomen is retracted with inspiration the midriff must be relatively immobile and is probably inflamed. This reversal of the abdominal wall movement is significant.

Psoas rigidity due to appendicitis or similar inflammatory condition in the vicinity is shown either by flexion of the corresponding thigh or by slight pain on moving the thigh. Slighter degrees may be detected by extending the thigh on the abdomen and noting if it causes pain.

It is seldom that the obturator muscle becomes rigid as the result of peritonitis, since the dense obturator fascia does not readily permit the inflammatory process to pass ; but occasionally putting the obturator muscle on the stretch by inverting the flexed thigh will elicit pain and denote some overlying inflamed focus which is penetrating and affecting the muscle.

There is another little test which may be of some value an fat subjects with a suspected inflammatory mass in the region of the iliac fossa and vessels. If the fingers be pressed on to the femoral artery in Scarpa's triangle so as to stop the circulation in the limb, the extra pulsation in the iliac vessels as the result of the block may cause an increase of pain when the inflammatory focus is in contact with the vessels. This femoral test would only be of real use in very fat people, who are notoriously difficult to investigate.

Gross anatomical derangements, with the exception of torsion of viscera and strangled external hernia, are not often obvious in the early stage of acute abdominal disease. The presence of free fluid and free gas displaces and masks the parts, but usually at too late a stage to be of much practical value.

\section{Conclusion}

The diagnosis of acute abdominal disease is a fascinating art which may be almost made into a science by attending carefully to the manifold symptoms to which it gives rise. The problems greatly differ from those in chronic abdominal disease: The combat against disease is in the one case a siege and the other a battle, and different qualities are required for success. In the acute disease early appreciation of the menacing forces is essential, and success may sometimes be gained by paying attention to small points which would in a chronic disease be regarded as trivial. In no other class of case is time of such importance, laboratory assistance of so little use, and the opportunity for acute clinical observation so well provided. Though disappointment comes but too often, the reward of paying attention to some of the neglected points which I have mentioned is an increasing number of successful diagnoses, and, what is even more important, an inward sense. of something logically attempted, something reasonably done.

\section{ACUTE OSTEOMYELITIS OF THE ILIUM BY}

\section{A. RENDLE SHORT, M.D., F.R.C.S. \\ SURGEON TO THE BRISTOL ROYAL INFIRMARY}

Probably it falls to the lot of most general surgeons at some time in their work to meet with this disease, but it is certainly rather uncommon, it presents a very difficult problem with regard to treatment, and there is extraordinarily little to be found about it in surgical literature. A considerable search for what may have been published on the subject during the past thirty years has yielded very meagre results. A case was reported by Murphy in 1916, secondary to furunculosis. A case of osteomyelitis of the ilium, probably tuberculous, was described in 1930 by Ryan and Funston. At a Russian surgical congress at Leningrad in 1925 Krasnobajev reviewed 428 cases of osteomyelitis treated by him in twenty years, with a general mortality of 22.4 per cent. There were twenty patients with osteomyelitis of the ilium-that is, about 5 per cent. of the whole number-and of these eight died. It is therefore more dangerous than osteomyelitis of the long bones. Two patients suffering from this disease have lately come under my care.

$$
\text { CASE I }
$$

Public school boy, aged 14. First seen July 19th, 1928, complaining of pain in right lumbar region attributed to a strain whilst diving two days before, when he "felt something go click." No physical signs, but limps a little.

July 20th.-Admitted to sanatorium. Has had no sleep on account of severe pain in buttock. Temperature $100^{\circ}$. Tender over right sacro-iliac joint. Temperature ran up to $102^{\circ}$, pulse 100 ; pain became very severe. Nepenthe gave no relief; no sleep.

July 21st.-Temperature $104.6^{\circ}$; very ill ; pain and tenderness persist. Operation under novocain. Dorsum ilii exposed but not trephined; periosteum scraped through. One doubtful spot of pus seen.

July 24th.-Pain has been better; wound not suppurating. Temperature $103^{\circ}-104^{\circ}$. Leucocytes 11,$000 ; 89$ per cent. polymorphonuclears. Mercurochrome given intravenously.

July 27th.-Abscess of right buttock opened. Culture: pure Staphylococcus aureus. 